\title{
FLEXIBLE MODELLING OF TIME TO FAILURE IN RISKY CAREERS
}

\author{
Scott E. Atkinson and John Tschirhart*
}

\begin{abstract}
Failure time models correcting for heterogeneity are used to explain the length of participation in a risky career. Using data from the National Football League, first we employ a class of techniques which ignore unobserved heterogeneity; hence these methods impose severe restrictions on the estimated hazard. We then examine a second class of techniques which correct for unobservables and thereby allow greater flexibility in the estimated hazard. Within this second class, we find that the estimated hazard using the Burr-12 density is much more accurate than densities in the first class, which include the exponential and Weibull. We expect that this density could be employed to successfully explain career duration in other high-risk, high-stress careers as well.
\end{abstract}

\section{Introduction}

$\mathrm{O}$ NLY recently, statistical models explaining duration data have been applied to explain the length of time in employment and non-employment spells and unemployment. ${ }^{1}$ In this paper we consider the characteristics of the set of models available to explain single spell employment data. Using these models, we then estimate determinants of labor duration in risky careers, where early retirement is common and immediate pensions may be available. Risky careers such as those found in police and fire protection, the military, air traffic control, and professional sports comprise an important segment of our labor force. Despite this, very little theoretical or empirical analysis has been performed on this sector. ${ }^{2}$

We determine the importance of covariates in explaining risky career duration with maximum likelihood estimation (MLE) of two classes of increasingly flexible density functions, both of

Received for publication May 13, 1985. Revision accepted for publication January 17, 1986.

* University of Georgia and University of Wyoming, respectively.

We wish to thank James Heckman, John Lapp, and anonymous referees for many useful comments, but we assume full responsibility for any errors.

${ }^{1}$ See Flinn and Heckman (1982a) on employment and nonemployment spells and see Butler and Worrall (1985), Flinn and Heckman (1982b), Kiefer and Neumann (1979), Nickell (1979), and Lancaster (1979) on unemployment.

${ }^{2}$ De Vany and Saving (1982) develop and test a theoretical model of demand and supply for entry level jobs in the Air Force. We refer to their work further below. Warner and Goldberg (1984) study reenlistments in the Navy to test how non-pecuniary factors influence retirement. Neither set of authors employs the time-to-failure methodologies used herein. which incorporate censoring. The first class of techniques postulates duration distributions which ignore the problem of omitted explanatory variables, i.e., unobserved heterogeneity. Within this class we estimate two parametric likelihoods based on the exponential and Weibull densities and the non-parametric likelihood of Cox (1972). Due to the potential bias from this procedure, we consider a second class of distributions called mixture distributions. We employ two procedures to construct continuous and finite mixtures.

We use these techniques to explain career duration with data on injuries, individual and team performance, education, race, and position for 260 National Football League (NFL) players active from 1971-80. We find that the Burr-12 continuous mixture distribution produces a far more accurate fit of the upside-down bathtub (UBT)shaped empirical hazard function than models which ignore unobservables, and that the finite mixture distributions we estimated were inferior. An UBT hazard over time typically describes accelerated life testing in which test items are subjected to abnormally high stress levels (Glaser, 1980). Consistent with our priors, the empirical analysis indicates that NFL players are subjected to a form of accelerated life testing.

The remainder of the paper is organized as follows. In part II the classes of duration models and their properties are developed. Data and results of the empirical estimation are presented in part III. The conclusion follows in part IV.

\section{Econometric Models}

Two basic approaches can be taken in explaining duration data with MLE methods. Under the first, the possibility of omitted regressors or unobserved heterogeneity is ignored. One then chooses among standard parametric densities such as the exponential or Weibull, or the non-parametric Cox (1972) marginal likelihood to characterize duration data. However, Lancaster (1979), Nickell (1979), and Heckman and Singer (1982, 1984) indicate that uncorrected heterogeneity results in 
biased coefficients, and in particular can result in negative duration dependence in models with no duration dependence at the individual level. That is, the estimated hazard falls more rapidly or rises less rapidly than it would under corrected heterogeneity.

Under the second approach, heterogeneity is controlled for, so that estimation can be made free of such heterogeneity bias. The only feasible method for single-spell data, such as duration in risky careers, is the random effect method (Heckman and Singer, 1982). We define a mixture probability density function (pfd) of $T$, where $T$ is a nonnegative, continuous random variable representing career length, as

$$
g(t)=\int f(t ; \theta) d H(\theta),
$$

where $f(t ; \theta)$ is the pdf of $T$ conditional on an unobserved heterogeneity component, $\theta$, an $m$ dimensional parameter vector whose cumulative distribution function (cdf) is $H(\theta)$ with pdf $h(\theta)$. $H$ is called the mixing distribution.

Heckman and Singer $(1982,1984)$ found that a more flexible "non-parametric" finite mixture of Weibull distributions is more consistent with theory than when normal, log normal, and gamma heterogeneity are combined with a Weibull density for duration times to form mixture densities. When $H$ is discrete and assigns positive probability to only a finite number of points $\left(\theta_{v}, v=\right.$ $1, \ldots, b)$, we replace the integral in (1) with a sum to yield a finite mixture

$$
g(t)=\sum_{v=1}^{b} H_{v}\left(\theta_{v}\right) f\left(t ; \theta_{v}\right) .
$$

Estimation of a mixture density in (1) requires choosing a continuous parametric distribution for the unobservables which is then mixed with $f(t ; \theta)$ by integrating out the random variables representing the unobservables. Estimation of a finite mixture in (2) requires choosing $b, H_{v}\left(\theta_{v}\right)$, and $f\left(t ; \theta_{v}\right)$. MLE is employed to estimate the parameters of both types of mixtures.

\section{A. Preliminaries}

Let all functions be defined over the interval $[0, \infty)$. Since $f(t)$ is defined as the pdf of $T$ in (1), the probability of an individual surviving until time $t$ is given by the survivor function

$$
S(t)=P(T \geq t)=\int_{t}^{\infty} f(x) d x,
$$

where $S(0)=1$ and $\lim _{t \rightarrow \infty} S(t)=0$. The pdf of $T$ is then

$$
f(t)=d(1-S(t)) / d t=-d S(t) / d t .
$$

The hazard function specifies the instantaneous rate of failure at $T=t$ conditional upon survival to time $t$ and is defined as

$$
\lambda(t)=f(t) / S(t) .
$$

With some manipulation, the pdf of $T$ can also be written as

$$
f(t)=\lambda(t) \exp \left(-\int_{0}^{t} \lambda(u) d u\right)
$$

\section{B. Uncorrected Heterogeneity}

A number of densities which ignore population heterogeneity have been used repeatedly to characterize failure times in the engineering and biostatistics literature (see Lawless, 1982). The Weibull pdf for time-to-failure of a career is

$$
f(t)=(c / \mu)(t / \mu)^{c-1} \exp \left[-(t / \mu)^{c}\right],
$$

where $\mu$ and $c$ are parameters affecting the scale and shape of $f(t)$, respectively. Now express $\ln \mu$ $=\gamma(\mathbf{z})$, so that $\mu=\exp [\gamma(\mathbf{z})]$ is a positive-valued function of $\mathbf{z}$, a $1 \times k$ vector of observations on $k$ covariates. If we let $\tilde{t}=\ln t, \sigma=c^{-1}$, and $\gamma(\mathbf{z})=$ $\mathbf{z} \boldsymbol{\beta}$, where $\boldsymbol{\beta}$ is an unknown $k \times 1$ vector of regression coefficients, we can write

$$
\tilde{t}=\mathbf{z} \boldsymbol{\beta}+\boldsymbol{\sigma} w,
$$

where $w$ has a standard extreme-value distribution with

$$
f(w)=\exp \left(w-e^{w}\right), \quad-\infty<w<\infty
$$

and

$$
S(w)=\exp \left(-e^{w}\right) .
$$

Least squares estimation of (8) is frequently inefficient compared to MLE methods. The likelihood of observing the log duration times for all $n$ individuals is

$$
L(\boldsymbol{\beta}, \boldsymbol{\sigma})=\prod_{i=1}^{n}\left[\boldsymbol{\sigma}^{-1} f\left(w_{i}\right)\right]^{\xi_{i}} S\left(w_{i}\right)^{1-\xi_{t}},
$$

where $\xi_{i}=0$ or 1 if the $i^{\text {th }}$ individual is censored or retires, respectively. 
The hazard function derived from (7) in terms of $t$ is

$$
\lambda(t)=(c / \mathbf{z} \boldsymbol{\beta})(t / \mathbf{z} \boldsymbol{\beta})^{c-1},
$$

which implies that the hazard is monotone increasing, constant, or decreasing over time for $c>1$, $c=1$, or $c<1$, respectively. To obtain the exponential density, let $\sigma=c^{-1}=1$ in (7)-(12). For the Weibull pdf in (7), expressing $\mu$ as a function of $\mathbf{z}$ independent of $t$, while $\sigma$ is independent of $\mathbf{z}$ and $t$, implies proportional hazards among durations of individual careers and a constant variance of the log duration of careers.

Parametric regressions involving Weibull or exponential proportional hazards models may make unwarranted assumptions regarding the hazard. The hazard may not be monotone increasing or decreasing as in the Weibull model or constant as in the exponential model. The Cox partially nonparametric proportional hazards model does not require that the hazard be monotone over time. Unfortunately, the benefit of this added flexibility, reduced bias relative to the more restrictive Weibull and exponential models, is accompanied by small to moderate decreases in efficiency. ${ }^{3}$

The Cox proportional hazards model specifies that

$$
\lambda\left(t_{i} ; \mathbf{z}_{i}\right)=\lambda_{0}(t) e^{\mathbf{z}_{i} \mathbf{\beta}},
$$

where $\lambda_{0}(t)$ is an arbitrary unspecified base-line hazard function for continuous $t$. The likelihood function for this model is given in Breslow (1974).

All three proportional hazards regression models can be generalized to time-dependent covariate models where the regression variables comprising $\mathbf{z}_{i}$ depend on time itself (see Lawless, 1982). Thus, the hazards of two events can vary in a non-proportional fashion over time, since the hazard becomes a function of time interacting with specified covariates.

\section{Correcting for Unobservables}

We correct for unobserved heterogeneity by first examining mixture densities and then finite mixtures. Many cdfs could be assumed for $H(\theta)$ in (1). We examine a mixture density of durations called the Beta-P, Singh-Maddala, or Burr-12

\footnotetext{
${ }^{3}$ See Lawless (1982).
}

which combines the Weibull pdf for $T$ with the gamma pdf for $\theta$, the latter of which is a very flexible density. ${ }^{4}$ The Burr- 12 pdf is computationally tractable since it yields closed form solutions for $f(t)$ and $S(t)$. From the Weibull pdf for $T$ in (7) we obtain

$$
f(t)=c \theta t^{c-1} \exp \left(-\theta t^{c}\right),
$$

where $\theta=\mu^{-c}$. Assuming $\theta$ has the gamma pdf

$$
h(\theta)=\delta^{p} \theta^{p-1} e^{\delta \theta} / \Gamma(p)
$$

where $\theta, p$, and $\delta$ are positive, we obtain from (1), after integrating out $\theta$, manipulating, and letting $\tilde{\delta}^{c}=\delta$

$$
g(t)=p c t^{c-1} \tilde{\delta}^{-c}\left[1+(t / \tilde{\delta})^{c}\right]^{-(p+1)}
$$

and

$$
G(t)=\left[1+(t / \tilde{\delta})^{c}\right]^{-p},
$$

where $G(t)$ is the survivor function..$^{5}$ To insure $\tilde{\delta}>0$, we let $\tilde{\delta}=\exp (\mathbf{z} \beta)$. The likelihood is constructed analogously to (11). The hazard function for the Burr-12 distribution can be derived from (16)-(17) as

$$
\begin{aligned}
\lambda(t) & =g(t) / G(t) \\
& =p c t^{c-1} \tilde{\delta}^{-c}\left[1+(t / \tilde{\delta})^{c}\right]^{-1},
\end{aligned}
$$

which is monotonically decreasing for $c \leq 1$ and assumes an UBT shape for $c>1$.

Finally, we examine finite mixtures under censoring. We first construct a mixture of Weibull pdf's for the log duration time, without imposing restrictions on parameters across mixtures. The likelihood for $n$ individuals is

$$
\begin{aligned}
L(\boldsymbol{\beta}, \boldsymbol{\sigma}, \boldsymbol{\theta})=\prod_{i=1}^{n} \prod_{v=1}^{b} & \left\{\sum _ { v } \theta _ { v } \left[\boldsymbol{\sigma}_{v}^{-1} f\left(w_{i} ; \boldsymbol{\beta}_{v}, \boldsymbol{\sigma}_{v}\right)^{\xi_{i}}\right.\right. \\
& \left.\left.+S\left(w_{i} ; \boldsymbol{\beta}_{v}, \sigma_{v}\right)^{1-\xi_{l}}\right]\right\},
\end{aligned}
$$

where the constraint $\Sigma \theta_{v}=1$ is imposed. Substituting (9) and (10) into (19) yields the Weibull finite mixture. The Burr-12 finite mixture is constructed analogously.

\footnotetext{
${ }^{4}$ See McDonald (1984). Also while the Burr-12 is consistent with a mixture, it does not imply a mixture.

${ }^{5}$ Details are available from the authors upon request.
} 


\section{Econometric Estimation}

\section{A. Data}

We estimate our career retirement models using a random sample of NFL players who entered the league between 1971 and 1980. Individuals still playing in 1981 were censored. A career in the NFL is both a risky and shortlived first career. During the 1970s, over fourteen thousand injuries were reported by the NFL, and the average career lasted 4.5 years.

Most studies of retirement have considered the final retirement decision of older workers and not the career-switching retirements often associated with risky careers. ${ }^{6}$ The literature suggests that the final retirement decision of older workers is usually a supply response brought about by the desire for more leisure, failing health, or the availability of pensions. However, retirement from the NFL may often be a demand response; that is, management may want to replace veteran players with potentially more productive, or lower salaried rookies. De Vany and Saving (1982) model the demand and supply for entry level jobs (i.e., first jobs) which is more applicable to our risky careers than the old-age retirement models. They develop a model of labor supply for entry level jobs and then embed this into an inventory model of employment demand. Equilibrium is achieved when the supply of workers and their duration of employment support the demand for workers.

To explain career length we select covariates that should be important in determining a player's decision to retire as well as management's decision to release a player. Data limitations do not allow us to distinguish between these separate, related decisions. Although professional football is a unique career due to high injury rates, heavy demands on physical skills, high visibility, and starting wages far in excess of opportunity wages, we find that the factors influencing retirement are ones that we would expect to have counterparts in more mundane, risky careers. The covariates used to explain failure times include:

$$
\begin{aligned}
P E R F= & \text { yards gained per attempt in the } \\
& \text { last year played divided by yards } \\
& \text { gained per attempt in all years; }
\end{aligned}
$$

\footnotetext{
${ }^{6}$ See, for example, Gordon and Blinder (1980).
}

$$
\begin{aligned}
P C T W I N S= & \text { weighted average percentage of } \\
& \text { games won by player's teams; } \\
D E G R E E= & \text { a binary variable }(1=\text { college } \\
& \text { degree received; } 0=\text { not re- } \\
& \text { ceived }) ; \\
L O P G= & \text { number of serious lower-body in- } \\
& \text { juries per game; } \\
R A C E= & \text { a binary variable }(1=\text { black; } 0 \\
& =\text { white }) ; \\
Q B= & \text { a binary variable }(1=\text { quarter- } \\
& \text { back, } \mathrm{QB} ; 0=\text { running back, } \mathrm{RB}, \\
& \text { and wide receiver, WR }) ; \text { and } \\
R A C E \cdot \quad & \text { an interaction term between } \\
D E G R E E= & R A C E \text { and } D E G R E E .
\end{aligned}
$$

The ratio of civilian to military salaries is a significant variable in explaining Air Force enlistments in the De Vany and Saving study. Salary should also be important in determining the length of NFL players' careers. However, in the absence of publicly available data on salaries before 1982 and no data on incentive payments, we employ $P E R F$ as a proxy, assuming that the more productive players were rewarded with higher salaries. Naturally, this would have been expected in a competitive environment where individuals receive their marginal revenue product, but NFL owners possessed substantial monopsony power. Nevertheless, Atkinson, Stanley, and Tschirhart (1985) using post-1981 data have found that while marginal revenue products exceed salaries, they have a Pearson correlation with performance of 0.6 that is statistically significant at the 0.01 level. By treating performance as an exogenous factor, we implicitly assume that players perform to their given abilities, subject to the performance of their teammates and opponents over which they have little influence.

Unfortunately, no uniform measure of performance exists across positions. Performance at many positions can be judged in terms of yards gained. However, data on offensive and defensive linemen's performance in blocking and neutralizing offensive linemen, respectively, are not available. Further, yards allowed on defense are usually assigned to the unit as a whole rather than any individual. Thus, we are limited to available data on yards gained by QBs, RBs, and WRs. The league-wide ratio of players at these positions in 
1980 was $60: 100: 100$. Thus, we sample randomly over the years 1971-80, selecting 260 NFL players -60 QBs, $100 \mathrm{RBs,}$ and $100 \mathrm{WRs}$ - who began their careers during this time period. Since $43 \%$ of our sample was censored in 1981 (still active) and only $57 \%$ had failed (retired), the censoring techniques examined above become critical.

To construct $P E R F$ we first obtained passing and running yardage per attempt for QBs, running yardage per attempt for RBs, and receiving yardage per attempt for WRs from various issues of the Football Register (FR). We then computed PERF as each player's yardage per attempt in his final year divided by his average yardage per attempt over his career. This measures the player's marginal performance relative to his expected performance, and should indicate whether retirement was influenced by waning marginal performance. Even though average yards per attempt may vary dramatically by position, $P E R F$ is unitless, so that $P E R F$ for all positions can be combined into a single variable. ${ }^{7}$ The expected influence of this variable, ceteris paribus, is that better performance increases a player's salary and utility derived from playing and, thereby, lengthens his career. A mitigating, but less important influence should be that superior performance enhances a player's salary in future careers through increased opportunities outside football, for example, as a television color commentator.

We compute PCTWINS as the weighted-average percentage wins of all teams played on, obtained from various issues of the Pro Football Weekly Almanac. All else equal, PCTWINS should enhance both a player's football salary and utility derived during his football career, hence, lengthening his career. Winning increases both the player's utility through added enthusiasm and comaraderie and his salary via playoff game bonuses and improved productivity.

We obtained data on the dummy variable $D E G R E E$ from various issues of $F R$. Although all NFL players in our sample attended college, only $35 \%$ received a bachelor's degree. ${ }^{8}$ The expected

\footnotetext{
${ }^{7}$ We experimented with other definitions of this variable. For example, we defined $P E R F$ as a player's yardage in his last year relative to the average of all players in that year and position. However, neither this nor our other formulations performed as well.

${ }^{8}$ According to the $1979 F R$, of 1,691 active players, 38\% received degrees.
}

effect of DEGREE is ambiguous. In a complex sport such as pro football, a degree may lengthen careers if it reflects greater intelligence and ambition. However, a degree may shorten football careers if it enhances post-football job opportunities. One argument is that the former influence should outweigh the latter so that college graduates experience longer careers. Ninety-six percent of their degrees did not allow entry into technical or professional occupations which are traditionally higher-salaried; only $4 \%$ were law, engineering, or graduate degrees. A counter-argument is that a degree allows entry into coaching and teaching in secondary schools and colleges, thereby shortening pro football careers.

$L O P G$ is computed as the total number of lower body injuries per game played throughout a player's career which resulted in being listed as "out" in the NFL Weekly Status Reports prior to each game. Injured players are reported in the Status Reports as being either "probable," "questionable," "doubtful," or "out" early in the week preceding a game. The "out" category is the most serious and means that the player cannot participate in the upcoming game. Lower body injuries include leg, hip, knee, ankle, foot, achilles tendon, and hamstring injuries, and accounted for $61 \%$ of all injuries to QBs, RBs, and WRs during the decade. In our sample, one serious "out" to the lower body occurred every 100 games, on average, with a standard deviation of 2.7 per 100 games. We expect serious injuries to shorten careers, all else equal, since a player's skills and physical abilities are reduced. Naturally, longer careers can be expected to produce more injuries; we are interested in whether players with a higher rate of serious lower body injuries, which reduce speed and quickness, will retire earlier. ${ }^{9}$

We examine the effect of $R A C E$, obtained from the NFL Player's Association (NFLPA) in Washington, D.C., since concern over racial discrimination in the NFL (with 54\% black players) prompted the NFLPA to commission a study of such practices in the NFL's management and coaching ranks (Braddock (1980)). If discrimina-

\footnotetext{
${ }^{9}$ We also examined an injury variable defined as "outs" per game played from injuries to the upper and lower body, which performed poorly. Including less serious injuries did not reduce the prob-value. These results agree with our priors that a high rate of serious leg injuries is more likely to degrade physical skills than upper body injuries.
} 
Table 1.-Test Statistics for Time to Failure Models

\begin{tabular}{|c|c|c|c|c|}
\hline & \multirow{2}{*}{$\begin{array}{c}\text { Cox } \\
\text { Proportional } \\
\text { Hazards }\end{array}$} & \multicolumn{3}{|c|}{ Log Likelihood for $f(t)^{\mathrm{a}}$} \\
\hline & & Weibull & Exponential & Burr Type-12 \\
\hline $\begin{array}{l}\text { Restricted } \log \\
\text { Likelihood }\left(\ln \Omega_{R}\right) \\
\text { for } \mathrm{H}_{0} \text { all slope } \\
\text { coefficients }=0\end{array}$ & -728.03 & -512.92 & -527.32 & -382.62 \\
\hline $\begin{array}{l}\text { Unrestricted Log } \\
\text { Likelihood }\left(\ln \Omega_{U}\right) \\
-2\left(\ln \Omega_{R}-\ln \Omega_{U}\right)\end{array}$ & $\begin{array}{r}-713.36 \\
29.33\end{array}$ & $\begin{array}{r}-491.86 \\
42.12\end{array}$ & $\begin{array}{r}-514.51 \\
25.62\end{array}$ & $\begin{array}{r}-366.16 \\
32.92\end{array}$ \\
\hline $\begin{array}{l}\text { Prob-Value for } \\
\text { Global } \chi^{2} \\
\text { Number of Observations }\end{array}$ & $\begin{array}{c}<0.005 \\
260\end{array}$ & $\begin{array}{c}<0.005 \\
260\end{array}$ & $\begin{array}{c}<0.005 \\
260\end{array}$ & $\begin{array}{c}<0.005 \\
260\end{array}$ \\
\hline
\end{tabular}

tion exists within the NFL so that blacks have fewer career-two opportunities in NFL coaching and/or if discrimination exists in the economy as a whole so that blacks have fewer career-two opportunities in all occupations, then, ceteris paribus, blacks should experience longer careers.

We obtain data on $Q B$ from various issues of $F R$. Typically, QBs require more on-the-job training to achieve a given level of proficiency than WRs or RBs. Thus, we include a $Q B$ dummy variable to account for the greater reluctance, ceteris paribus, to cut veteran QBs as their skills and performance wane, due to greater initial training and development costs for replacements.

Finally, a wide disparity exists regarding the degree status of black and white players. Only $14 \%$ of the players in our sample are blacks who earned a degree. This implies that only $26 \%$ of the blacks in our sample earned a degree, in contrast to $46 \%$ of the whites. Thus, we include an interaction term, DEGREE $\cdot R A C E$, to account for the possibility that a degree may be worth less to a black than to a white player, ceteris paribus. ${ }^{10}$

\section{B. Estimation}

The results for the Cox, Weibull, exponential, and Burr-12 proportional hazards models are re-

\footnotetext{
${ }^{10}$ We examined other interaction terms, including PERF and PCTWINS with all the other variables, but found them not to be significant at standard levels. We also experimented with time dependent covariates for PCTWINS, LOPG, and $P E R F$, with similar results for the Cox, Weibull, and exponential models.
}

ported in tables $1-3 .^{11}$ Estimated coefficients for all models were obtained using Newton-Raphson algorithms which employ analytical first and second-order partial derivatives. ${ }^{12}$ For all models, convergence was rapid and results were insensitive to a wide range of starting values. ${ }^{13}$

We carry out our tests of functional form for nested densities and tests of the null hypothesis $\left(\mathrm{H}_{0}\right)$ that all slope coefficients are zero (termed the global chi-square test), for each model using $-2 \ln \lambda$, where $\lambda$ equals the ratio of the maximum of the restricted likelihood function $\left(\Omega_{R}\right)$ to the maximum of the unrestricted likelihood function $\left(\Omega_{U}\right)$. This statistic is asymptotically distributed as $\chi_{\hat{r}}^{2}$ where $\hat{r}$ is the number of restrictions.

Based on the global $\chi^{2}$ in table 1, we reject the $\mathrm{H}_{0}$ above for all models (all prob-values $<0.005$ ) and proceed to judge among density functions. Comparing the unrestricted Weibull and exponential likelihood functions, $-2 \ln \lambda=45.30$ with one degree of freedom. Thus, we reject the null hypothesis that $\sigma=1$ (i.e., that the exponential regression is an acceptable specialization of the Weibull), with prob-value less than 0.005 . As displayed in table 2, the Weibull suggests that since

\footnotetext{
${ }^{11}$ We also estimated ordinary least squares (OLS), since it is simpler than MLE. A priori we reject OLS based on its inability to incorporate censoring and its substantially different likelihood specifications. The coefficients of this model differed dramatically from those of the other four models.

${ }_{12}$ To assure the accuracy of these programs, numerous results of Kalbfleisch and Prentice (1980) were replicated for the Cox, Weibull, and exponential models. All computations were carried out on a CDC Cyber 760 computer.

${ }^{13}$ The convergence criteria was that the change in each parameter value was $<0.001$ times its value.
} 
Table 2. - Estimated Parameters for Proportional Hazards Time to Failure Models (ASYMPTOTIC $t$-VALUES IN PARENTHESES)

\begin{tabular}{|c|c|c|c|c|}
\hline & \multirow{2}{*}{$\begin{array}{c}\text { Cox } \\
\text { Proportional } \\
\text { Hazards }(-\boldsymbol{\beta})\end{array}$} & \multicolumn{3}{|c|}{ Dependent variable $=\ln (t)$} \\
\hline & & Weibull & Exponential & Burr Type-12 \\
\hline \multicolumn{5}{|l|}{ Independent Variables } \\
\hline Intercept & - & $\begin{array}{c}0.5856^{\mathrm{a}} \\
(2.9247)\end{array}$ & $\begin{array}{c}0.5851^{b} \\
(1.8866)\end{array}$ & $\begin{array}{c}0.0045 \\
(0.0133)\end{array}$ \\
\hline PCTWINS & $\begin{array}{r}1.7071^{\mathrm{a}} \\
(3.1398)\end{array}$ & $\begin{array}{r}1.4134^{a} \\
(4.0757)\end{array}$ & $\begin{array}{r}1.5128^{a} \\
(2.8984)\end{array}$ & $\begin{array}{r}1.0698^{a} \\
(3.1778)\end{array}$ \\
\hline$R A C E$ & $\begin{array}{c}0.4700^{\mathrm{a}} \\
(2.0694)\end{array}$ & $\begin{array}{r}0.3475^{\mathrm{a}} \\
(2.4745)\end{array}$ & $\begin{array}{c}0.4393 \\
(1.9448)\end{array}$ & $\begin{array}{c}0.3616^{\mathrm{a}} \\
(2.2388)\end{array}$ \\
\hline$Q B$ & $\begin{array}{c}0.4979^{\mathrm{a}} \\
(2.0176)\end{array}$ & $\begin{array}{r}0.3961^{\mathrm{a}} \\
(2.6132)\end{array}$ & $\begin{array}{r}0.4763^{\mathrm{b}} \\
(1.9583)\end{array}$ & $\begin{array}{c}0.2289 \\
(1.4474)\end{array}$ \\
\hline$D E G R E E$ & $\begin{array}{r}0.6778^{\mathrm{a}} \\
(2.6552)\end{array}$ & $\begin{array}{c}0.5105^{\mathrm{a}} \\
(3.2822)\end{array}$ & $\begin{array}{c}0.6119^{\mathrm{a}} \\
(2.4269)\end{array}$ & $\begin{array}{r}0.6455^{\mathrm{a}} \\
(3.6681)\end{array}$ \\
\hline$R A C E \cdot D E G R E E$ & $\begin{array}{l}-0.9780^{\mathrm{a}} \\
(-2.7689)\end{array}$ & $\begin{array}{l}-0.7483^{\mathrm{a}} \\
(-3.4870)\end{array}$ & $\begin{array}{l}-0.9059^{\mathrm{a}} \\
(-2.6009)\end{array}$ & $\begin{array}{l}-0.6828^{\mathrm{a}} \\
(-2.8767)\end{array}$ \\
\hline$L O P G$ & $\begin{array}{l}-4.8188^{b} \\
(-1.6503)\end{array}$ & $\begin{array}{l}-3.2394^{\mathrm{b}} \\
(-1.7059)\end{array}$ & $\begin{array}{c}-4.4958 \\
(-1.5798)\end{array}$ & $\begin{array}{c}-2.2584 \\
(-1.2177)\end{array}$ \\
\hline PERF & $\begin{array}{c}0.0674 \\
(0.5156)\end{array}$ & $\begin{array}{c}0.0671 \\
(0.8113)\end{array}$ & $\begin{array}{c}0.0703 \\
(0.5338)\end{array}$ & $\begin{array}{c}0.0916 \\
(1.2954)\end{array}$ \\
\hline Shape Parameters o & & $\begin{array}{r}0.6158^{a} \\
(15.6538)\end{array}$ & $1.0^{\mathrm{c}}$ & \\
\hline$c$ & & & & $\begin{array}{r}2.5564^{a} \\
(5.6303)\end{array}$ \\
\hline$p$ & & & & $\begin{array}{c}0.5691^{\mathrm{a}} \\
(2.0748)\end{array}$ \\
\hline
\end{tabular}

a Significant at the 0.05 level using a two-tailed test. ${ }^{b}$ Significant at the 0.05 level using a one-tailed test. c Constrained to equal 1.0 .

$\sigma=p^{-1}<1$ the hazard increases over time, which is intuitively plausible for such a physically punishing occupation as professional football. However, further investigation indicates that this conclusion is premature.

The log likelihood functions for the Burr-12 are greater than those of the Weibull, and both shape parameters for the Burr-12 are statistically significant as shown in table 2. We suspect that a substantial number of covariates are unobservable for NFL players. Moreover, we believe a com-

Table 3.-Sample Average Estimated Hazard Functions

\begin{tabular}{cccc}
\hline \hline$t$ (years) & Weibull & Burr-12 & $\begin{array}{c}\text { Empirical } \\
\text { Hazard }\end{array}$ \\
\hline 1 & 0.1404 & 0.1512 & 0.1577 \\
2 & 0.2163 & 0.2635 & 0.1684 \\
3 & 0.2786 & 0.2827 & 0.2214 \\
4 & 0.3334 & 0.2648 & 0.1939 \\
5 & 0.3832 & 0.2379 & 0.1754 \\
6 & 0.4294 & 0.2118 & 0.1053 \\
7 & 0.4727 & 0.1890 & 0.1852 \\
8 & 0.5138 & 0.1696 & 0.2353 \\
9 & 0.5529 & 0.1534 & 0.1250 \\
\hline
\end{tabular}

pelling argument can be made for the superiority of one model over another by comparing the estimated hazard functions to the empirical hazard. This is done in table 3 where we present the population average estimated hazard functions, $t=1, \ldots, 9$, for the Weibull and Burr-12 models and the empirical hazard. The latter is computed as $d_{t} / r_{t}$, where $d_{t}$ is the number of retirements in period $t$ and $r_{t}$ is the number at risk in time period $t$, equal to the number of players active and uncensored in period $t-1$. Judging the two estimated parametric hazards on their closeness to the empirical hazard, the Burr-12 is clearly preferable based on a $\chi^{2}$ test of goodness of fit. Its UBT shape corresponds far more closely to the UBT empirical hazard, particularly after $t=3$, where both the Burr-12 and empirical hazards turn down. Since the Weibull hazard rises monotonically, it increasingly overestimates the hazard beyond this point.

The empirical hazard exhibits a smaller UBT shape for duration of 7-9 years. Thus, we fit a two-component finite mixture of Weibull pdfs and 
then of Burr-12 pdfs to the data in hopes of improving on the fit of the single Burr- 12 pdf. We imposed no equality restrictions on slope or scale coefficients across the mixtures. However, both alternatives performed poorly. Each Weibull component exhibited strongly increasing hazards, fitting the empirical hazard poorly based on a $\chi^{2}$ test. Because the careers of only 27 players lasted from 7-10 years, shape parameters of the second Burr-12 mixture component were highly insignificant. Thus, the single Burr- 12 pdf was clearly superior among the simple pdfs, the mixture densities, and the finite mixtures examined, based on significance of shape parameters and $\chi^{2}$ tests of goodness of fit of the empirical hazard. Direct comparison to the Cox model is precluded, since it does not employ a pdf.

Table 2 displays the estimated covariate coefficients and their asymptotic standard errors for the four models. Because of our strong expectations regarding the signs of the coefficients for $P E R F$, $P C T W I N S, L O P G$, and $Q B$, we carry out a one-tailed test at the 0.05 level. All signs are as expected; however, only the coefficient on PCTWINS is significant across all models. The $Q B$ coefficient is significant in all but the Burr-12 model, while the $L O P G$ parameter is significant in the Cox and Weibull models only. PERF's coefficient is not significant in any of the models. Although PERF, $Q B$ and $L O P G$ possess insignificant coefficients in the Burr-12 model at the 0.05 level, they are significant at approximately the 0.10 level using a one-tailed test.

Since our priors for DEGREE, RACE, and $R A C E \cdot D E G R E E$ are inconclusive, we employ two-tailed tests of their coefficients at the 0.05 level. The coefficient for $R A C E$ is positive and significant in all models except the exponential where it is nearly so. The coefficient on DEGREE is positive and significant for all models; and the interaction term between race and degree $(R A C E$ - DEGREE) is negative and significant for all models. From the magnitudes of these three coefficients in the Burr-12 model, we can draw the following conclusions, ceteris paribus. Blacks without degrees tend to play longer than whites without degrees, but blacks with degrees tend to have shorter careers than whites with degrees. This does not support the claims of racial discrimination in football. We also conclude that whites with degrees have longer careers than whites without degrees, and blacks with degrees have shorter careers than blacks without degrees. Thus, the value of a degree within football is inconclusive.

We calculated "beta coefficients" for both models where each coefficient measures the effect on duration of equally likely changes in its covariate. In the preferred Burr-12 model, DEGREE, RACE - DEGREE, PCTWINS, and RACE rank in decreasing order of importance in explaining the log of career length. $Q B$ and $L O P G$ rank next, while PERF places last, about one fifth as important as DEGREE. ${ }^{14}$

Finally, our results suggest that the extreme life testing model is appropriate. Rookies experience an increasing hazard for their first several years. This occurs presumably because many new players, after an early grace period, cannot absorb the physical punishment and perform to the increasingly strict standards required, and they retire or are cut at an increasing rate. Players who survive the middle "shakeout" years experience a falling hazard for the remainder of their careers.

\section{Conclusion}

We explain the length of participation in a risky career using techniques which ignore unobserved heterogeneity and ones which correct for unobservables, thereby allowing greater flexibility in the estimated hazard. Our expectation was that careers in a physically demanding and punishing career such as professional football should exhibit an UBT-shaped hazard found with extreme life testing. The Burr-12 mixture density, whose hazard takes on this shape, yiélded an estimated hazard which was much more accurate than that for models where unobserved heterogeneity was ignored. Additionally, the finite mixture densities proved inferior. The Burr-12 distribution should also be successful in explaining retirements from other high-risk, high-stress careers.

\section{REFERENCES}

Atkinson, Scott E., Linda R. Stanley, and John Tschirhart, "Evidence of Non Profit-Maximizing Firms: Revenue Sharing and Factor Payments in the NFL," University of Wyoming working paper, April 1985.

Braddock, J. H., Institutional Discrimination: A Study of

${ }^{14}$ Elasticities of $T$ with respect to each covariate can be derived for the Weibull and Burr-12 models. See McDonald (1984). Computed elasticities are omitted here since they add little additional information. 
Managerial Recruitment in Professional Football, Report prepared for the National Football League Players Association (1980).

Breslow, N. E., "Covariance Analysis of Censored Survival Data," Biometrics 30 (1974), 89-99.

Butler, Richard J., and John D. Worrall, "Work Injury Compensation and the Duration of Nonwork Spells," Economic Journal 95 (Sept. 1985), 714-724.

Cox, D. R., "Regression Models and Life Tables (with discussion)," Journal of the Royal Statistical Society, B, 34 (1972), 187-220.

De Vany, Arthur S., and Thomas R. Saving, "Life-Cycle Job Choice and the Demand and Supply of Entry Level Jobs: Some Evidence from the Air Force," this REVIEW 64 (Aug. 1982), 457-465.

Flinn, C., and James Heckman, "Models for the Analysis of Labor Force Dynamics," in Robert Basmann and George Rhodes (eds.), Advances in Econometrics, Vol. 1 (Greenwich, CT: JAI Press, 1982a).

, "New Methods for Analyzing Structural Models of Labor Force Dynamics," Journal of Econometrics 18 (Jan. 1982b), 115-168.

Glaser, Ronald E., "Bathtub and Related Failure Rate Characterizations," Journal of the American Statistical Association 75 (Sept. 1980), 667-672.

Gordon, Roger H., and Alan S. Blinder, "Market Wages, Reservation Wages, and Retirement Decisions," Journal of Public Economics 14 (Oct. 1980), 277-308.

Heckman, James J., and Burton Singer, "The Identification Problem in Econometric Models for Duration Data," in Advances in Econometrics, Proceedings of World Meet- ings of the Econometric Society (1980) (Cambridge: Cambridge University Press, 1982), 39-77.

"A Method for Minimizing the Impact of Distributional Assumptions in Econometric Models for Duration Data," Econometrica 52 (Mar. 1984), 271-320.

Kalbfieisch, J. D., and R. L. Prentice, The Statistical Analysis of Failure Time Data (New York: John Wiley and Sons, 1980).

Kiefer, Nicholas, and George Neumann, "An Empirical Job Search Model with a Test of the Constant Reservation Wage Hypothesis," Journal of Political Economy 87 (Feb. 1979), 69-82.

Lancaster, Tony, "Econometric Methods for Duration of Unemployment," Econometrica 47 (July 1979), 939-956.

Lawless, J. F., Statistical Models and Methods for Lifetime Data (New York: John Wiley, 1982).

McDonald, James B., "Some Generalized Functions for the Size Distribution of Income," Econometrica 52 (May 1984), 647-664.

National Football League, National Football League Weekly Status Report (New York: 1971-1980).

Nickell, Stephen, "Estimating the Probability of Leaving Unemployment," Econometrica 47 (Sept. 1979), 1249-1266.

Pro Football Weekly, Inc., Pro Football Weekly Almanac (Chicago, 1972-1981).

The Sporting News, Football Register, 1971-1981 Editions (St. Louis, 1971-1981)

Warner, John T., and Matthew S. Goldberg, "The Influence of Non-pecuniary Factors on Labor Supply: The Case of Navy Enlisted Personnel," this REview 66 (Feb. 1984), $26-35$. 